\title{
Shi Pi Yin Herbal Decoction
}

National Cancer Institute

\section{Source}

National Cancer Institute. Shi Pi Yin Herbal Decoction. NCI Thesaurus. Code C125632.

A traditional Chinese medicine (TCM) decoction containing a mixture of Aconiti lateral root, Zing iberis (ginger) root, Fu Ling (Poria), Atractylodis macrocephalae, Chaenomelis fruit (flowering quince fruit), magnolia bark, Aucklandia costus root, Arecae pericarpium, Tsao-Ko (cardamon) fruit, and licorice root, and may also contain Arecae seeds and Ziziphus jujuba fruit (red date), which may be used to treat edema. Although the exact mechanism(s) of action through which Shi Pi Yin decoction exerts its effect has yet to be fully elucidated, upon oral administration, this TCM may reduce edema. 